and evolved (Fig. 1). Carrying out single-cell DNA sequencing at different times during this experiment confirmed that there was ongoing chromosomal instability.

It has previously been difficult to assess the mechanisms that generate this instability in a native environment. Bolhaqueiro and colleagues' organoid model is as close a representation of that as is so far possible. The authors noted that most of the chromosomepartitioning errors arose from the formation of chromatin bridges, in which a chromosome, or part of one, is stretched between the two separating masses of cellular DNA at the final stage of cell division. Such errors are indicative of structural changes during chromosome replication that occur before cell division, and have been observed in 2D monolayers of colorectal cancer cells ${ }^{8}$.

There is much debate about the cause of chromosomal instability in cancer. Chromatin bridges could arise as a result of other defects in cell-division processes, and further in-depth analyses using organoid models to investigate the specific nature of such chromosomepartitioning defects and any other abnormal cell-division processes might shed light on this.

A subset of human colorectal cancers do not have the usual hallmarks of chromosomal instability, and although they have a high level of nucleotide mutations (termed microsatellite instability), they have normal or almost normal chromosome numbers, and there is little chromosomal difference between cells. Bolhaqueiro et al. made the surprising discovery that some organoids of this subtype of colorectal cancer also have appreciable rates of chromosomal errors during cell division. DNA sequencing of single cells obtained from clinical samples of tumour tissues by Bolhaqueiro and colleagues revealed that chromosomal instability can occur along with microsatellite instability in the same tumour. The low level of chromosomal instability in these tumours probably hindered its detection in earlier bulk DNA-sequencing methods that did not use a single-cell sequencing approach.

Bolhaqueiro et al. also noted that, for each individual organoid, there was only a weak correlation between the number of times that cells made errors in chromosomal partitioning during cell division and the level of variation in chromosome numbers between cells in the organoid. This discrepancy would make sense if there were differences in how some organoids respond to chromosomal-partitioning errors. Live-cell imaging indeed revealed that organoids with a high variability in chromosome number were better able to withstand errors during cell division than were organoids that had low chromosome-number variability. If such errors occurred in organoids that had low variability in chromosome number, then cell division often stopped or the cells died.

These results suggest that chromosome-number variability in tumour cells is the product of the rate of development of chromosomal instability and the ability of cells of a given tumour type to tolerate further changes in chromosome number or structure $^{11}$. The factors that govern whether or not a tumour will tolerate aneuploidy remain mostly unknown. A better understanding of this would probably reveal potential targets for the development of new therapies.

As the use of organoid model systems becomes more widespread, experiments that can directly compare the results obtained in 3D and $2 \mathrm{D}$ cultures would help to ensure that the appropriate culture systems are chosen for the specific research question and experimental approach involved. This would allow moredirect comparisons between the results from different groups. With regard to Bolhaqueiro and colleagues' work, it would be interesting to know whether the rate of chromosomal instability and aneuploidy observed would have been different had 2D growth systems been used.

It would also be helpful to know how representative previous work in $2 \mathrm{D}$ monolayer systems is of what happens in vivo, and which questions might need to be re-evaluated using organoids. For example, although a normal 3D tissue architecture might be essential for preventing chromosomal instability during cell division in non-cancer cells, it is possible that, if cancer cells have already lost the normal checkpoints to combat faulty cell-division processes, such cells will exhibit similar rates and mechanisms of chromosomal instability regardless of whether they are grown in 2D or
3D systems. If so, organoid studies might be needed only to confirm those carried out in 2D systems, rather than being essential.

Finally, aneuploidy is emerging as a possible early event driving tumour evolution ${ }^{12,13}$, but information about the frequency and types of chromosomal instability during the initial stages of tumour evolution is extremely limited. If organoids could be used to examine chromosomal instability during these preliminary stages, or to study abnormal cells (called precursor lesions) that might be on a pathway to forming cancer, this would undoubtedly yield many fascinating insights.

Sarah C. Johnson and Sarah E. McClelland are at the Barts Cancer Institute, Queen Mary University of London, London EC1M 6BQ, UK. e-mail:s.mcclelland@qmul.ac.uk

1. Bolhaqueiro, A. C. F. et al. Nature Genet. 51, 824-834 (2019).

2. Lengauer, C., Kinzler, K. W. \& Vogelstein, B. Nature 386, 623-627 (1997).

3. Taylor, A. M. et al. Cancer Cell 33, 676-689 (2018).

4. Hieronymus, H. et al. eLife 7, e37294 (2018).

5. Bakhoum, S. F., Danilova, O. V., Kaur, P., Levy, N. B. \& Compton, D. A. Clin. Cancer Res. 17, 7704-7711 (2011).

6. Sachs, N. \& Clevers, H. Curr. Opin. Genet. Dev. 24 , 68-73 (2014).

7. Ertych, N. et al. Nature Cell Biol. 16, 779-791 (2014)

8. Burrell, R. A. et al. Nature 494, 492-496 (2013).

9. Kopper, O. et al. Nature Med. 25, 838-849 (2019).

10. Knouse, K. A., Lopez, K. E., Bachofner, M. \& Amon, A. Cell 175, 200-211 (2018).

11. McClelland, S. E., Burrell, R. A. \& Swanton, C. Cell Cycle 8, 3262-3266 (2009).

12.Cross, W. et al. Nature Ecol. Evol. 2, 1661-1672 (2018) 13. Teixeira, V. H. et al. Nature Med. 25, 517-525 (2019)

This article was published online on 3 June 2019.

\title{
Ozone mystery laid to rest
}

Measurements of atmospheric ozone levels taken during the nineteenth century cast doubt on the computational models used today to simulate the atmosphere. An independent proxy of past ozone levels offers reassurance. SEE LETTER P.224

\section{MATHEW EVANS}

B efore the 1950s, direct observations of the composition of the atmosphere were extremely limited. Air trapped in pockets of snow and ice has allowed the observational record for some gases to be extended back hundreds of years. But certain gases that affect climate and air quality, such as ozone $\left(\mathrm{O}_{3}\right)$, are not stable in ice or snow, limiting their records to the past few decades. On page 224, Yeung et al. ${ }^{1}$ report that isotopic observations of oxygen $\left(\mathrm{O}_{2}\right)$ molecules trapped in polar snow and ice can provide a new constraint on estimates of ozone levels in the troposphere (the lowest 12 kilometres of the atmosphere) over the past 150 years. This greatly extends our knowledge of the concentration of this key atmospheric gas, and might finally address a problem that has worried atmospheric chemists for decades.

Advances in atmospheric science are often made by taking advantage of the 'experiments of opportunity' that occur as a result of natural and human-driven changes to the atmosphere. It is therefore crucial to make long-term measurements of the atmosphere. The modern era for such measurements began in the late 1950s, with observations of carbon dioxide levels ${ }^{2}$ from the Mauna Loa Observatory in 


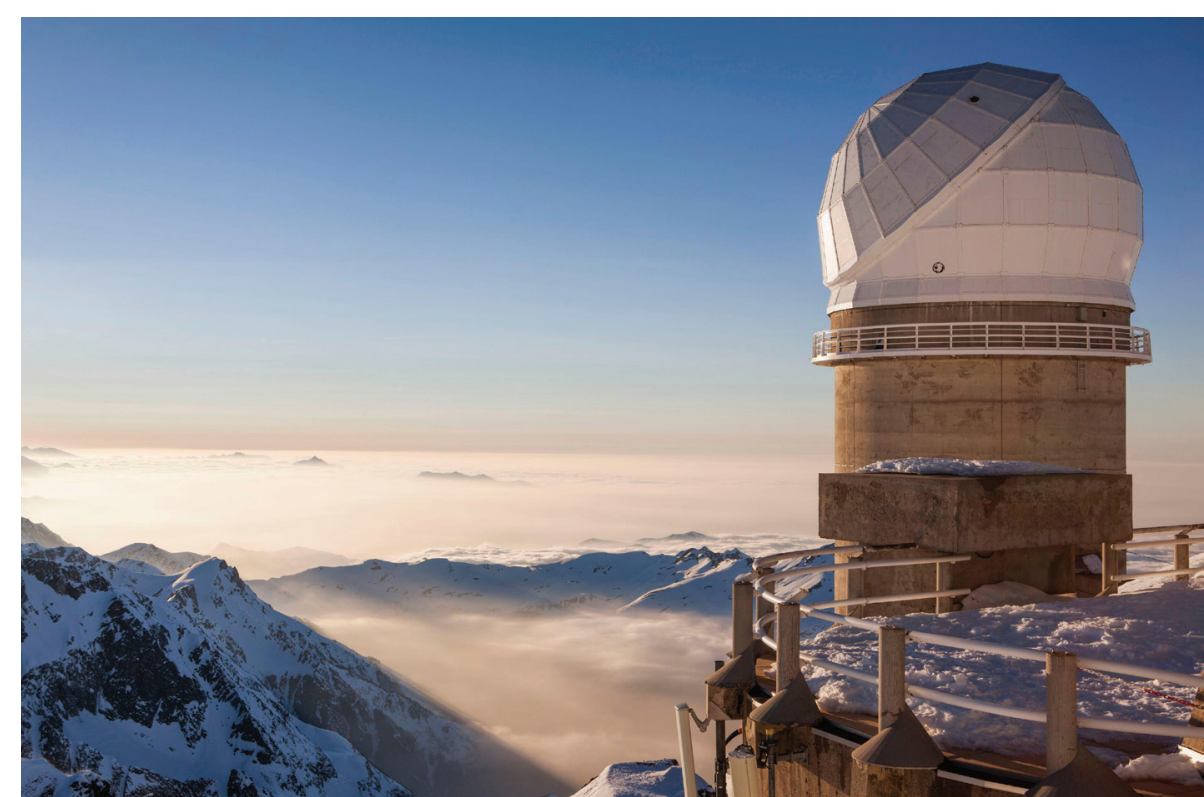

Figure 1 | The Pic du Midi Observatory, France. Measurements of atmospheric ozone levels were made during the nineteenth century, including some at the Pic du Midi Observatory. Yeung et al. ${ }^{1}$ report an independent constraint on atmospheric ozone levels that suggests the concentrations recorded in the nineteenth century were artificially lowered by other pollutants.

Hawaii. Long-term measurements of other atmospheric components started soon after that. It was only in the 1990s that observational networks expanded sufficiently to provide a global perspective of a wide range of the air's components ${ }^{3}$.

However, the impact of the atmosphere's changing composition depends on both the present-day concentrations of pollutants and their concentrations before the change started. Many atmospheric changes (climate change being the most notable example) are associated with the Industrial Revolution, and so, to understand the magnitude of those effects, we need to understand the composition of the atmosphere over the past 100-200 years.

The changes in concentrations of some components of the air, such as $\mathrm{CO}_{2}$ and methane, are known quite well from the ice record and from modern observations. However, because the ice record provides no constraints on estimates of changes in ozone levels, computer models of atmospheric chemistry and physics are used instead. These models calculate a roughly $40 \%$ increase in tropospheric ozone levels between 1850 and the present ${ }^{4}$. The Intergovernmental Panel on Climate Change uses these estimates to evaluate the relative importance of ozone as a greenhouse gas, compared with $\mathrm{CO}_{2}$ and methane, and thereby to develop climate policy ${ }^{5}$.

The use of computational estimates of ozone wouldn't be a concern if it weren't for an unlikely set of ozone measurements made in the nineteenth century by scientists at several sites around the world (Fig. 1). These measurements were made using various techniques that have since been assessed by present-day scientists - who calibrated the data to place the results on modern scales and worked out that other pollutants, such as sulfur dioxide, could have interfered with the measurements ${ }^{6,7}$. It was difficult, however, to assess the magnitude of such interference. Surprisingly, the data suggested that nineteenth-century ozone concentrations were very low, and increased by about $300 \%$ during the industrial period - a much larger change than was calculated by models ${ }^{8}$.

On the face of it, these results have enormous implications. Given that present-day concentrations of ozone calculated by models are roughly correct ${ }^{9}$, a failure to simulate concentrations before the industrial period would suggest that there is a fundamental problem with our understanding of atmospheric chemistry. In that case, how could we trust the models' predictions of future atmospheric composition, and therefore formulate climate policy? If ozone concentrations had increased much more than was previously thought, then the role of the gas in climate change would be larger than had been assumed, and so efforts to lower ozone levels would have greater potential to reduce global warming.

It seemed implausible to most atmospheric chemists that the computer models could be so wrong. The most likely explanation was that sulfur dioxide and other chemical reductants produced by coal burning during the Industrial Revolution interfered with the observational techniques used at that time. But the absence of an independent observational constraint on historical ozone levels has meant that the nineteenth-century observations have vexed atmospheric chemists for the past few decades.

Although ozone molecules do not remain trapped in ice and snow, oxygen molecules do. Yeung et al. therefore measured the amounts of the common oxygen-16 isotope and of the much less common oxygen-18 isotope in oxygen molecules trapped in polar ice and snow.

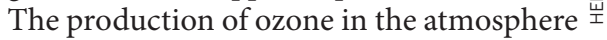
changes the proportion of these isotopes in atmospheric oxygen molecules. The isotopic record of trapped oxygen molecules therefore contains a history of ozone concentrations over the past 150 years.

Yeung and colleagues' analysis shows that the increase in tropospheric ozone over that period was around $40 \%$, much smaller than the increases indicated by the nineteenth-century observations, and consistent with the numbers predicted by models. It therefore seems likely that interference from sulfur dioxide and other gases had indeed artificially lowered the ozone concentrations recorded in the historical measurements.

Those nagging doubts of atmospheric chemists can probably now be laid to rest. Perhaps disappointingly for some, there doesn't seem to be a fundamental problem with our understanding of atmospheric chemistry, which means that the scope of ozone management in helping to reduce climate change is limited: the radiative forcing (greenhouse warming) caused by tropospheric ozone is only around $22 \%$ of that caused by $\mathrm{CO}_{2}$ (ref. 10 ). However, efforts to reduce tropospheric ozone concentrations shouldn't stop. Every little helps in the fight against climate change, and reductions would help to prevent some of the approximately one million deaths estimated to be caused by tropospheric ozone each year ${ }^{11}$.

Other mysteries remain in atmospheric chemistry, including the sources of atmospheric organic aerosols, the chemistry of halogen-containing molecules, and how naturally occurring emissions interact with those associated with human activities. But Victorian scientists don't have much to say about any of those.

Mathew Evans is at the Wolfson

Atmospheric Chemistry Laboratories,

Department of Chemistry, University of York,

York YO10 5DD, UK.

e-mail:mat.evans@york.ac.uk

1. Yeung, L. Y. et al. Nature 570, 224-227 (2019).

2. Keeling, C. D. Tellus 12, 200-203 (1960).

3. Schultz, M. G. et al. Elementa 3, 000067 (2015).

4. Young, P. J. et al. Atmos. Chem. Phys. 13, 2063-2090 (2013)

5. Intergovernmental Panel on Climate Change. Climate Change 2014: Synthesis Report (IPCC, 2014).

6. Volz, A. \& Kley, D. Nature 332, 240-242 (1988)

7. Marenco, A., Gouget, H., Nédélec, P.,

Pagés, J.-P. \& Karcher, F. J. Geophys. Res. Atmos. 99, 16617-16632 (1994).

8. Mickley, L. J., Jacob, D. J. \& Rind, D. J. Geophys. Res. Atmos. 106, 3389-3399 (2001).

9. Young, P. J. et al. Elementa 6, 10 (2018).

10. Myhre, G. et al. in Climate Change 2013: The Physical Science Basis. Contribution of Working Group I to the Fifth Assessment Report of the Intergovernmental Panel on Climate Change (eds Stocker, T. F. et al.) Ch. 8, 659-740 (Cambridge Univ. Press, 2013).

11. Malley, C. S. et al. Environ. Health Perspect. 125, 087021 (2017) 